\title{
Progress of Mucocutaneous Leishmaniasis to Drug Nonresponsive Diffuse Cutaneous Leishmaniasis in Ethiopia. A Case Report
}

This article was published in the following Dove Press journal: International Medical Case Reports Journal

\author{
Banchamlak Tegegne' \\ Getaneh Alemu (D) ${ }^{2}$ \\ 'Amhara Public Health Institute, Bahir \\ Dar, Ethiopia; ${ }^{2}$ Department of Medical \\ Laboratory Science, Bahir Dar University, \\ Bahir Dar, Ethiopia
}

Background: Leishmaniasis is one of the neglected tropical diseases, yet it is associated with high mortality and morbidity. It is caused by intracellular protozoan parasites of the genus Leishmania. Based on the infecting parasite species and host factors, leishmaniasis could be manifested as cutaneous (localized or diffuse), mucocutaneous or visceral clinical forms. In Ethiopia, L. aethiopica is well known to cause all forms of cutaneous leishmaniasis, the localized form being mostcommon.

Case Presentation: An adult patient from Yayu district, west Oromia region of Ethiopia presents with multiple skin lesions on his face, hands and mutilation of nose through both nostrils. Eight years before the present symptoms, he has developed spontaneously healed cutaneous ulcer on his face leaving a permanent scar. Physical examination revealed multiple nodular lesions on his hands, face and nose as well as swelling of the upper lip. Parasitological examination of ulcer lesion revealed presence of amastigotes, and the patient was diagnosed with mucocutaneous leishmaniasis. He was treated with sodium stibogluconate $(20 \mathrm{mg} / \mathrm{kg} /$ day IM for 30 days) and clinically cured. After two years, he presented with clinical outcomes typical of diffuse cutaneous leishmaniasis. After confirmation by parasitological examination, he was put on sodium stibogluconate $(20 \mathrm{mg} / \mathrm{kg} /$ day IM for 30 days $)$ and paromomycin (15 mg/kg/day IM for 30 days) combination therapy. As he showed no progress, he was treated with prolonged sodium stibogluconate $(20 \mathrm{mg} / \mathrm{kg} / \mathrm{day}$ IM for 60 days) monotherapy. He was still nonresponsive and discharged uncured.

Conclusion: The present case is unusual in Ethiopia with relapse of mucocutaneous leishmaniasis progressing to diffuse form. The later form was nonresponsive for both mono and combination therapy, therefore, formulating new drugs or evaluating other antiLeishmania drugs is required.

Keywords: mucocutaneous leishmaniasis, diffuse cutaneous leishmaniasis, case report

\section{Background}

Leishmaniases refers to clinical syndromes caused by obligate intracellular protozoan parasites of the genus Leishmania. ${ }^{1,2}$ The clinical outcome depends on the virulent characteristics of the infecting parasites and the cell-mediated immune responses of hosts. More often, specific Leishmania species are associated with a typical clinical picture. ${ }^{3}$ Some species are primarily dermatotrophic to cause cutaneous leishmaniasis (CL), while others are mainly viscerotropic to cause visceral leishmaniasis (VL). Leishmania donovani complex causes VL. ${ }^{4}$ L. major, L. tropica and L. aethiopica cause CL in the old world while L. mexicana,
$\mathrm{PO}$ Box 79

Tel +251922842776

Email getanehmlt@gmail.com
International Medical Case Reports Journal 2020:13 55I-555 
L. amazoniensis, L. guyanensis, L. panamensis and L. braziliensis are the main etiologic agents of CL in the new world. ${ }^{5}$ However, considerable overlap exists in that the relationship between the clinical syndromes and infecting species is not always as distinct as in the case of L. aethiopica and L. infantum. ${ }^{6}$

Cutaneous leishmaniasis is manifested as localized cutaneous leishmaniasis (LCL), diffuse cutaneous leishmaniasis (DCL), and mucocutaneous leishmaniasis (MCL). MCL develops due to extension of local skin disease into the mucosal tissue while DCL covers a wide area of the skin without involvement of the mucosa. ${ }^{7}$ Oral and nasal mucosa are frequently affected in MCL, with rare involvement of the laryngeal and pharyngeal mucosa. ${ }^{8}$ Both MCL and DCL develop from primary LCL via direct extension, bloodstream or lymphatics. There is also the possibility of MCL development if the sand fly bites in the transition between skin and mucosa. ${ }^{7}$ Ethiopia is endemic for localized form of CL. However, countable numbers of cases with MCL and DCL also exist in the country, all forms primarily caused by L. aethiopica. ${ }^{3,6}$ Both MCL and DCL are less-responsive to available anti-leishmanial drugs, and often, relapse after discontinuation of treatment. ${ }^{9}$ However, it was unusual to see a change in clinical forms from MCL to DCL during relapse. Hence, we presented a case report of a patient who developed DCL two years after the primary MCL healed with treatment.

\section{Ethics Approval and Consent to Participate}

No institutional approval was required to publish this case report. Written informed consent was obtained from the patient with reference number 1073/2020.

\section{Case Presentation}

A 38-year-old male patient visited the outpatient department of Felege Hiwot Specialized Referral Hospital, Bahir Dar City, Northwest Ethiopia. He presented with multiple skin lesions on his face, hands and mutilation of nose as shown in Figure 1. His upper lip was injured and swollen. He reported that cutaneous ulcers started eight years before on both his cheeks and subsided by itself leaving di-pigmented scars. He originally lived in Senbo village of Achilo Kebele, Yayu district, Illubabor zone, Oromia region of Western Ethiopia. He has no history of alcohol abuse, chronic diseases, HIV or immunomodulatory drug use. He said that eight years before it started as a small

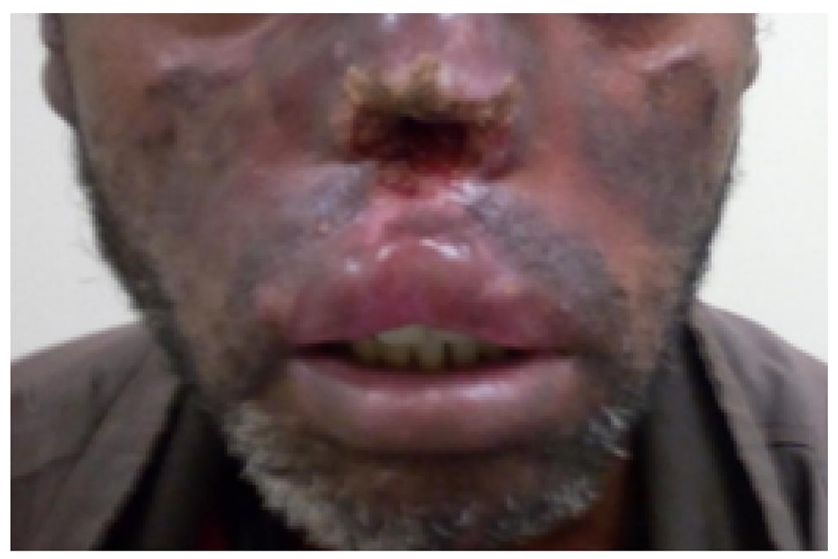

Figure I MCL. Affecting the nose, upper lips and face.

papule on his right check and progressed to an open ulcer. Gradually, ulcers progressed to the left cheek and the forehead. Active lesions lasted for about six months and healed spontaneously leaving permanent scars.

During healing, the ulcers started to dry/crust at the center and proceed to the periphery. The present manifestation started at two sites (the edge of the nasal openings and right hand). As shown in Figure 2, later on, the ulcer disseminated to cover both hands and mutilate the nose through both nostrils. However, there was no blockage of the nasal openings. Physical examination revealed multiple nodular lesions on his hands, face and nose as well as swelling of the upper lip. Ulcers were crusted at the center and have raised contours at the periphery. The lesions were infected. Multiple healed scars were observed on his face mainly on both cheeks implying previous infection. No systemic signs and symptoms like fever, swelling of liver and/or spleen and lymphedema were seen.

Leishmaniasis was suspected and he was sent to Amhara Public Health Institute, Bahir Dar City for parasitological investigation. Skin lesions were collected aseptically from nose, face and hands, and processed for parasitological examination. A smear was prepared directly from the

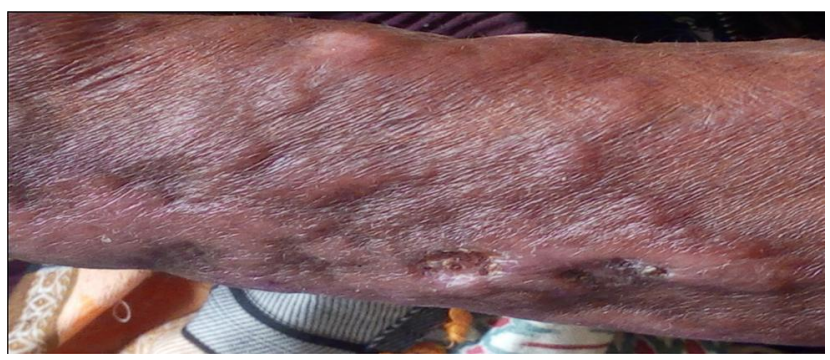

Figure 2 Disseminated CL. Affecting hands with characteristic multiple nodular lesions which crust at the center. 


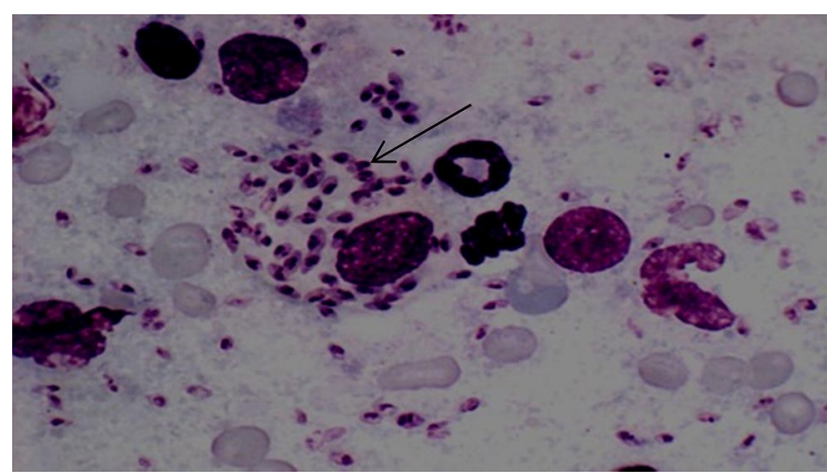

Figure 3 Amastigotes from Giemsa-stained smear prepared from skin lesion.

lesions and examined microscopically after staining with Giemsa. Skin sample was also cultured in NovyMacNeal-Nicolle culture media; however, because the ulcers were infected, the culture was contaminated and reliable results were not obtained. As shown in Figure 3, microscopic examination of the stained smear revealed presence of many macrophages filled with amastigote stages of Leishmania graded as $+6 .{ }^{10}$ Based on nonreactive rK39 rapid diagnostic test result and absence of related signs and symptoms VL was ruled out. Therefore, based on the clinical manifestations and smear result, the patient was diagnosed with MCL. He was referred to Addisalem hospital, the nearest hospital where leishmania treatment service is available, to start the therapy.

He was admitted and treated with sodium stibogluconate (SSG) (20 mg/kg/day IM for 30 days) and a broad spectrum antibiotic. He was clinically cured and discharged. Two years later, he presented with a quite different cutaneous manifestation from the previous one. As shown in Figure 4, he presented with multiple, nonpitting, closed papules on his face, nose hands and legs. He reported that the present manifestation started to appear in the last three months and was preceded by intense itching.

Skin smear was examined using microscopically and culture techniques. Intramacrophage amastigotes were detected from the smear with a parasite load grade of +6 and the culture was also positive for promastigotes. Hence, he was diagnosed with DCL. He was initially treated with cloxacillin $(500 \mathrm{mg}$ qid for five days), SSG (20 mg/kg/day IM for 30 days) and paromomycin (15 mg/kg/day IM for 30 days). Wound care was also given twice a day for four days. He showed no improvement after four weeks of treatment and was put on prolonged treatment with SSG (20 mg/kg/day IM for 60 days). Still there was no improvement and re-examination of skin sample revealed presence of amastigotes. Since he was not responsive to the available anti-leishmanial drugs, he was requested to start amphotericin B treatment. However, he was not willing to do so; instead he strongly asked to be discharged. In respect of the patient's decision, he was discharged uncured.

\section{Discussion and Conclusions}

Cutaneous leishmaniasis has been known in Ethiopia since 1913. ${ }^{11}$ Sand fly vectors transmitting CL are mainly found in the altitude ranges of 1400-2700 mabove sea level, thus limiting the distribution of $\mathrm{CL}$ to the Ethiopian highlands. ${ }^{12,13}$ Therefore, about 30 million people are at risk of infection, of which 20,000-40,000 are infected every year. ${ }^{3,14}$ Yayu district, where the present case lives, is located at an average altitude of $1788 \mathrm{~m}$ above sea level, making the area conducive for CL transmission.

L. aethiopica is responsible for about $99 \%$ of CL cases in Ethiopia. ${ }^{15}$ It is a pleiotropic species and has different genetic multiplicity, that diverse clinical forms could be manifested based on the infecting strain. ${ }^{6,16}$ However, in Ethiopia, LCL

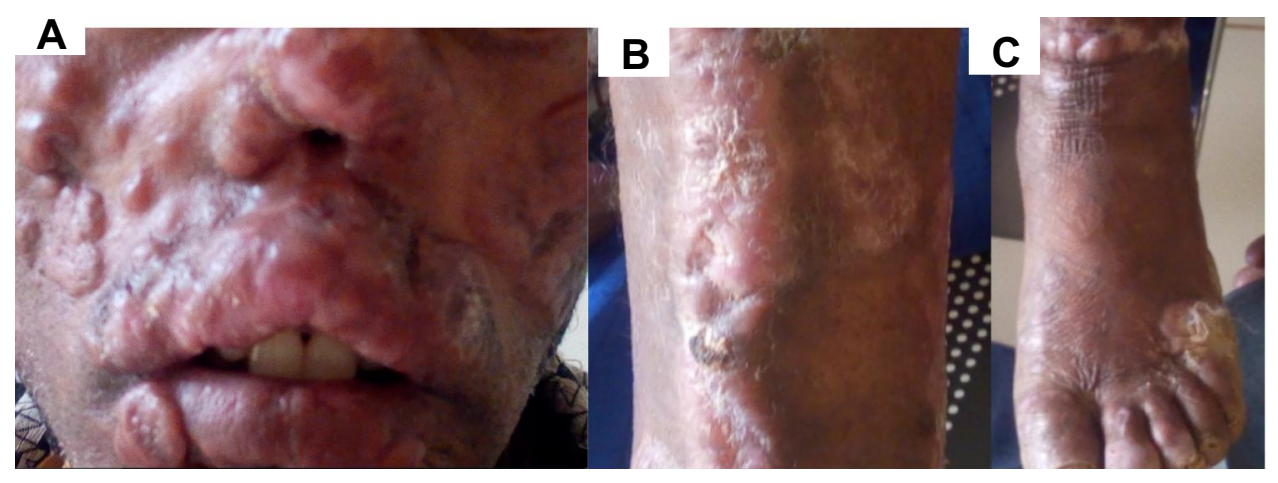

Figure $4 \mathrm{DCL}$. Affecting the face (A) and leg (B and $\mathbf{C})$. 
is the most frequent manifestation compared to MCL and DCL. ${ }^{16}$ LCL usually presents on the face (90\%) and extremities, and starts as a small red papule and widens to measure up to $2 \mathrm{~cm}$ in diameter to form a nodule or plaque. Lesions spontaneously heal within three to six months leaving a permanent scar. ${ }^{10}$ In the present case study, we cannot be sure about the etiology of spontaneously healed ulcer which occurred eight years prior. However, based on the characteristics of the ulcer and scars, there could be a strong suspicion of CL.

Mucocutaneous leishmaniasis occurs when the sand flies inoculate promastigotes on the mucosal border of nose and mouth, or due to spread of parasites from nearby cutaneous lesions via direct extension, bloodstream or lymphatics. $^{7,10}$ In Ethiopia, MCL is usually caused by L. aethiopica and the manifestation is limited to the mouth and nose. MCL may destruct and disfigure the nose and sometimes perforate the nostrils to result in tapir nose. Unlike LCL, MCL do not spontaneously heal and it needs treatment. ${ }^{10} \mathrm{SSG}$ has been ineffective for the treatment of patients with MCL in Ethiopia. Despite this the present case was treated with SSG, possibly due to unavailability of alternative drugs with better efficacy.

Despite the fact that all forms of CL are mainly caused by the same species, it is uncommon to see a relapse of MCL that manifests as DCL. ${ }^{17}$ This might be evidence for increasing recent reports of DCL in the old world. ${ }^{15}$ DCL could be with or without mucosal involvement and most of the time do not respond to treatment. ${ }^{18,19}$ DCL is expected among immunosuppressed patients. However, there are reports of DCL among immunocompetent patients pronouncing the potential of certain parasite strains to disseminate in healthy individuals. ${ }^{20}$ In the present report, we were unable to evaluate the immune status of the patient due to lack of laboratory logistics; but his medical record showed that he has no history of chronic diseases like HIV, tuberculosis, cancer and he did not take any immunosuppressive drugs. The national guideline for treatment of DCL recommends combination therapy with paromomycin and SSG in a synergistic dose of $10 \mathrm{mg} / \mathrm{kg} /$ day. ${ }^{10}$ However, in the present case, no improvement was seen after combination therapy for 30 days.

In the present case report, we were unable to rule out leprosy through skin slit examination. Hematological and immunological profile of the patient was not assessed, all due to lack of laboratory facility. Most importantly, we did not identify the species and strain of the parasite during development of each form of clinical presentation. This would have been important to differentiate between relapse and re-infection.

In conclusion, we have presented an atypical case of $\mathrm{CL}$ in Ethiopia with sequential clinical presentations of MCL and DCL. The later presentation was nonresponsive to both SSG monotherapy and SSG and paromomycin combination therapy. This calls for urgency in formulating and evaluating efficacious drugs for the treatment of disseminated forms of CL. Health professionals should also be aware of the presence of DCL and MCL in Ethiopia so that they can contribute for early diagnosis and treatment before the disease disfigures patients.

\section{Abbreviations}

CL, cutaneous leishmaniasis; DCL, diffuse cutaneous leishmaniasis; LCL, localized cutaneous leishmaniasis; MCL, mucocutaneous leishmaniasis; SSG, sodium stibogluconate; VL, visceral leishmaniasis.

\section{Data Sharing Statement}

The original data for this study is available from the corresponding author.

\section{Consent for Publication}

Written consent was obtained from the patient to publish his personal data and pictures or photos.

\section{Acknowledgments}

We thank Ateka Malede and Tsedalu Alemu for their help in getting the patient card and arranging a schedule to meet with the patient.

\section{Author Contributions}

Both authors contributed to data analysis, drafting or revising the article, have agreed on the journal to which the article will be submitted, gave final approval of the version to be published, and agree to be accountable for all aspects of the work.

\section{Disclosure}

The authors report no conflicts of interest in this work.

\section{References}

1. Mcgwire BS, Satoskar AR. Leishmaniasis: clinical syndromes and treatment. QJ Med. 2013;107(1):7-14. doi:10.1093/qjmed/hct116

2. Ngure PK, Kimutai A, Rukunga G, Tonui WK. A review of leishmaniasis in Eastern Africa. $J$ Nanjing Med Univ. 2009;23(2):79-86. doi:10.1016/S1007-4376(09)60032-0 
3. Alvar J, Velez ID, Bern C, et al. Leishmaniasis worldwide and global estimates of its incidence. PLoS One. 2012;7(5):e35671. doi:10.1371/ journal.pone.0035671

4. Agersew A, Alemu A, Nuraini E, et al. Knowledge, attitude and practices related to visceral Leishmaniasis among residents in Adis Zemen town, South Gondar, North West Ethiopia. BMC Public Health. 2013;13:382-388.

5. Clayton R, Grabczynska S. Mucocutaneous leishmaniasis presenting as facial cellulitis. $J$ Laryngol Otol. 2005;119(7):567-569. doi:10.1258/0022215054352252

6. Pratlong F, Dereure J, Ravel C, et al. Geographical distribution and epidemiological features of old world cutaneous leishmaniasis foci, based on the isoenzyme analysis of 1048 strains. Trop Med Int Health. 2009;14(9):1071-1085. doi:10.1111/j.1365-3156.2009. 02336.x

7. Pereira Diniz JL, da Rocha Costa MO, Goncalves DU. Braz. J Otorhinolaryngol. 2011;77(3):380-384.

8. Marsden PD. Mucosal leishmaniasis due to Leishmania (Viannia) Braziliensis in Tres Bracos, Bahia - Brazil. Rev Soc Bras Med Trop. 1994;27(2):93-101. doi:10.1590/S0037-86821994000200007

9. Reithinger R, Dujardin JC, Louzir H, Pirmez C, Alexander B, Brooker S. Cutaneous leishmaniasis. Lancet Infect Dis. 2007;7 (9):581-596. doi:10.1016/S1473-3099(07)70209-8

10. Federal Ministry of Health. Guideline for Diagnosis, Treatment and Prevention of Leishmaniasis in Ethiopia. 2nded.; 2013.

11. Ashford RW, Bray MA, Hutchinson MP, Bray RS. The epidemiology of cutaneous leishmaniasis in Ethiopia. Trans $R$ Soc Trop Med Hyg. 1973;67(4):568-602.

12. Ashford R. The comparative ecology of Leishmania aethiopica. Ecology des Leishmanioses France:(Colloques Internationaux du CNRS No 239); 1977:233-240.
13. Lemma A, Foster W, Gemetchu T, Preston P, Bryceson A, Minter D. Studies on leishmaniasis in Ethiopia: I.-preliminary investigations into the epidemiology of cutaneous leishmaniasis in the highlands. Ann Trop Med Parasitol. 1969;63(4):455-472. doi:10.1080/ 00034983.1969 .11686649

14. Seid A, Gadisa E, Tsegaw T, et al. (2014) Risk map for cutaneous leishmaniasis in Ethiopia based on environmental factors as revealed by geographical information systems and statistics. Geospat Health. 2014;8(2):377-387. doi:10.4081/gh.2014.27

15. AHRI. Proceedings of the international consultative meeting on cutaneous leismaniasis in Ethiopia, Addis Ababa; July 4-5, 2011.

16. Padovese V, Terranova M, Toma L, Barnabas GA, Morrone A. Cutaneous and mucocutaneous leishmaniasis in Tigray, northern Ethiopia: clinical aspects and therapeutic concerns. Trans $R$ Soc Trop Med Hyg. 2009;103(7):707-711. doi:10.1016/j.trstmh.2009. 02.023

17. Seyed A, Alireza B, Sadaf S, Ali N, Mohammad S. First case report of atypical disseminated cutaneous leishmaniasis in an opium abuser in Iran. Rev Inst Med Trop Sao Paulo. 2018;60. doi:10.1590/S16789946201860005.

18. Vélez ID, Jiménez A, Vásquez D, Robledo SM. Disseminated cutaneous leishmaniasis in Colombia: report of 27 cases. Case Rep Dermatol. 2015;7(3):275-286. doi:10.1159/000441120

19. Hashiguchi Y, Gomez EL, Kato H, Martini LR, Velez LN, Uezato H. Diffuse and disseminated cutaneous leishmaniasis: clinical cases experienced in Ecuador and a brief review. Trop Med Health. 2016;44(1):2. doi:10.1186/s41182-016-0002-0

20. Hajjaran H, Mohebali M, Akhavan AA, Taheri A, Barikbin B, Soheila NS. Unusual presentation of disseminated cutaneous leishmaniasis due to Leishmania major: case reports of four Iranian patients. Asian Pac J Trop Med. 2013;6(4):333-336. doi:10.1016/ S1995-7645(13)60066-8
International Medical Case Reports Journal

\section{Publish your work in this journal}

The International Medical Case Reports Journal is an international, peer-reviewed open-access journal publishing original case reports from all medical specialties. Previously unpublished medical posters are also accepted relating to any area of clinical or preclinical science. Submissions should not normally exceed 2,000 words or 4

\section{Dovepress}

published pages including figures, diagrams and references. The manuscript management system is completely online and includes a very quick and fair peer-review system, which is all easy to use. Visit http://www.dovepress.com/testimonials.php to read real quotes from published authors. 